\title{
A Hybrid GA/GE Beamforming Technique for Side Lobe Cancellation of Linear Antenna
} Arrays

\author{
Amr H. Hussein ${ }^{*}$, Safaa I. Abd Elrahman ${ }^{2}$, and Abd Elhameed A. Shaalan ${ }^{3}$ \\ 1 Electronics and Electrical Communications Engineering Dept., Faculty of Engineering, Tanta University, Tanta, Egypt. \\ ${ }^{2,3}$ Electronics and Communication Engineering Dept., Faculty of Engineering, Zagazig University, Zagazig, Egypt. \\ Corresponding author, mail: amr.abdallah@f-eng.tanta.edu.eg, amrvips@yahoo.com
}

\begin{abstract}
Side lobe level reduction is one of the most critical research topics in antenna arrays beamforming as it mitigates the interfering and jamming signals. In this paper, a hybrid combination between the Genetic algorithm (GA) optimization technique and the gauss elimination (GE) equation solving technique is utilized for the introduction of the proposed GA/GE beamforming technique for linear antenna arrays. The GA is chosen as it is a search-driven optimization strategy based on the concepts of genetic and natural selection. It is often used to identify optimal or near-optimal solutions to complex problems that would otherwise take a very long time to solve. The proposed technique estimates the optimum excitation coefficients and the non-uniform inter-elements spacing for a specific side lobe (SL) cancellation without disturbing the half power beamwidth (HPBW) of the main beam. Different size Chebychev linear antenna arrays are taken as simulation targets. The simulation results revealed the effectiveness of the proposed technique. Furthermore, the feasibility of realistic validations of synthesized arrays is demonstrated using the computer simulation technology (CST) microwave studio software package, which provides users with an optimized modeling environment and results in realizable and realistic designs.
\end{abstract}

INDEX TERMS Genetic algorithm (GA), Particle swarm optimization (PSO), Side lobe level (SLL), and Uniform linear array (ULA)

\section{INTRODUCTION}

SLL reduction has a significant effect on the performance of wireless communication systems such as radars as it minimizes the received signal to interference ratio (SIR) and plays effective role in anti-jamming process. Many research efforts are exerted in this issue. In [1], a SLL reduction algorithm of uniform linear antenna arrays has been introduced. It utilized both the convolution procedure and time scaling propriety to determine the optimum elements excitations required to reduce the array SLL. But, the SLL is reduced at the expense of half power beamwidth broadening. In [2], an optimization technique based on particle swarm optimization (PSO) has been introduced for side lobe level reduction, half power beamwidth minimization, and pattern nulls control. The PSO searches for the optimum elements spacing and weights required to achieve these targets. In [3], a flower pollination algorithm (FPA) has been introduced for SLL reduction and pattern nulling of linear antenna arrays through controlling the amplitude or position of array element. But it is time consuming as it requires large number of iterations. In [4], the invasive weed optimization algorithm (IWO) for side lobe reduction of linear and circular antenna arrays has been introduced. The IWO has better performance in terms of accuracy, convergence, and stability compared to a biogeography based optimization algorithm (BBO), cuckoo search algorithm (CS), and the firefly algorithm (FA), and PSO.

A position-only optimization technique using the Gravitational Search Algorithm (GSA) has been introduced in [5] for pattern nulling and SLL reduction of uniform linear and planer antenna arrays. It provided higher performance than the PSO, Comprehensive Learning Particle Swarm Optimization (CLPSO) [6], Ant Colony Optimization (ACO) [7], Spider Monkey Optimization (SMO) [8], and Self-adaptive Hybrid Differential Evolution algorithm (SHDE) [8]. But it takes thousands number of iterations to obtain the results. And in case of SLL reduction, it reduces only the first side lobe rather than the reduction of the entire side lobes.

In [9], a SLL reduction technique of linear and planar antenna arrays based on Grey Wolf (GWO) and Imperialist Competitive (ICA) optimization algorithms has been introduced. It performs array thinning for SLL reduction by setting some of the elements excitations to zero or turn off the selected antenna element. The array thinning is performed using GWO and ICA optimization techniques. In [10], the cuckoo search (CS) algorithm is used for synthesis of linear antenna arrays by controlling the amplitude, phase, and position of the array elements to reduce the SLL with and 
without arbitrary null placement. The simulation results showed the simplicity and the effectiveness of the CS algorithm. In [11], a new adaptive beamforming (ABF) algorithm for side lobe suppression by placing extra radiation pattern nulls has been introduced. It is utilized to steer the main lobe towards a desired signal and place radiation pattern nulls towards respective interference signals to achieve a desired SLL. The algorithm has introduced a relatively high SLL reduction but at the expense of HPBW broadening.

In [12], side lobe level reduction of linear arrays by using unequally spaced elements is introduced. For simplicity and faster design realization, the array factor is converted into a triangular system of linear equations which can be easily solved using a recursive algorithm. The results showed that this method has more accuracy and speed than the other analytical methods. It also provides lower SLL, simulation time and an improvement of $3 \mathrm{~dB}$ beamwidth when compared to equally spaced array. In [13], a gravitational search algorithm (GSA) is presented for $\mathrm{ABF}$ in linear antenna arrays with multi-objective function in order to reduce the SLL and direct the main lobe towards desired user and make nulls towards the interfering signals.

In this paper, a GA optimization based beamforming technique for side lobe (SL) cancellation of linear antenna arrays without disturbing the half power beamwidth (HPBW) of the main beam is introduced. The proposed technique attempts to determine the optimum excitation coefficients and the non-uniform elements spacing required to cancel a specific SL to mitigate the unintended interferers. The GA is chosen as it is a search-driven optimization strategy based on the concepts of genetic and natural selection. It is often used to identify optimal or near-optimal solutions to complex problems that would otherwise take a very long time to solve. The key difference between the GA and the other optimization techniques such as PSO is that GA is initially a discrete technique that is often ideal for combinatorial problems, while PSO is a continuous technique that is extremely unsuitable for combinatorial problems. This multi-parent effect can have a significant advantage over single-point optimization techniques like tabu search, and simulated annealing.

\section{Proposed SL Cancellation Technique}

In this section, a GA optimization based beamforming technique for a specific side lobe cancellation is introduced. It is based on the simple GE equation solving technique and the GA optimization. This hybrid combination between the GA and GE results in a relatively fast beamforming technique. The GA searches for the optimum non-equal elements spacing while the corresponding excitations are calculated by solving a set of deterministic equations whose derivation is expressed below. Consider a uniform linear array consisting of $M$ antenna elements with uniform element spacing $d$, its array factor $A F(\theta)$ is given by [14]:

$$
\mathrm{AF}(\theta)=\sum_{\mathrm{m}=1}^{\mathrm{M}} \mathrm{a}_{\mathrm{m}} \mathrm{e}^{\mathrm{j}(\mathrm{m}-1) \mathrm{kd} \cos \theta}
$$

where $a_{m}$ is the excitation coefficient of the $m^{\text {th }}$ antenna elements, $k=2 \pi / \lambda$ is the wave number, $\lambda$ is the wavelength. The proposed technique follows these steps:

\section{A. Shaping Function Generation}

Firstly, the uniform array pattern is generated according to (1). Then the left and right null angles $\theta_{l n}$ and $\theta_{r n}$ of the intended $n^{\text {th }}$ side lobe are determined as shown in Fig. 1 where $M=10$ elements broadside Chebychev linear array is considered. Taking the first side lobe as example, then $\theta_{l 1}=$ $107.7^{\circ}$ and $\theta_{r 1}=115.2^{\circ}$.

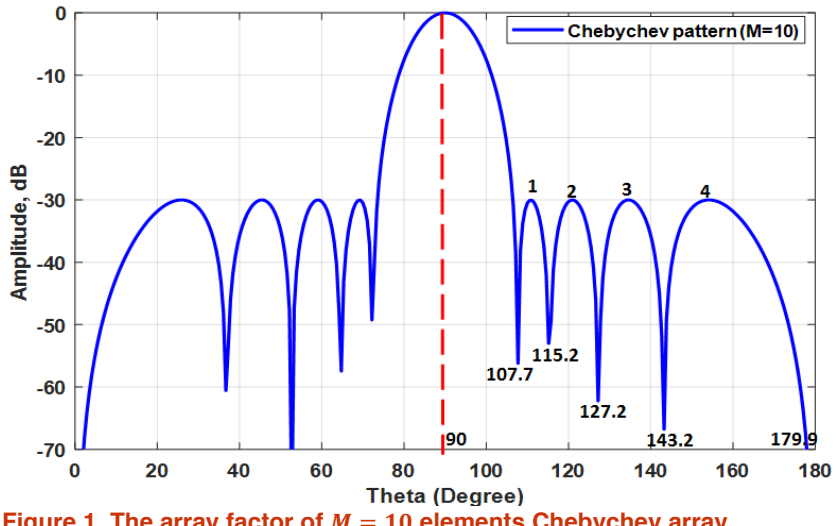

Figure 1. The array factor of $M=10$ elements Chebychev array.

Then, the $n^{\text {th }}$ symmetrical side lobes to be cancelled are forced to zero by multiplying the array pattern $A F(\theta)$ by a generated shaping function $f_{s h}(\theta)$. The shaping function consists of two shifted rectangular functions of the same width $\tau_{n}=\left(\theta_{r n}-\theta_{l n}\right)$ where $\theta_{r n}>\theta_{l n}$. For broadside array, the right shifted rectangular function $f_{r}(\theta)$ can be expressed as:

$$
f_{r}(\theta)=\operatorname{rect}\left\{\frac{\theta-\theta_{R n}}{\tau_{n}}\right\}
$$

where $\theta_{R n}=\frac{\theta_{r n}+\theta_{l n}}{2}$ is the center angle of the $n^{\text {th }}$ right side lobe. While the center angle of the $n^{\text {th }}$ left side lobe is given by:

$$
\theta_{L n}=90^{\circ}-\left(\theta_{R n}-90^{\circ}\right)=\left(180^{\circ}-\theta_{R n}\right)
$$

Then the left rectangular function $f_{l}(\theta)$ is shifted by $\theta_{L n}$ and can be expressed as:

$$
f_{l}(\theta)=\operatorname{rect}\left\{\frac{\theta-\theta_{L n}}{\tau_{n}}\right\}=\operatorname{rect}\left\{\frac{\theta-\left(180^{\circ}-\theta_{R n}\right)}{\tau_{n}}\right\}
$$

From (2) and (4), the shaping function $f_{s h}(\theta)$ shown in Fig. 2 is given by:

$$
f_{s h}(\theta)=1-\left(f_{l}(\theta)+f_{r}(\theta)\right)
$$




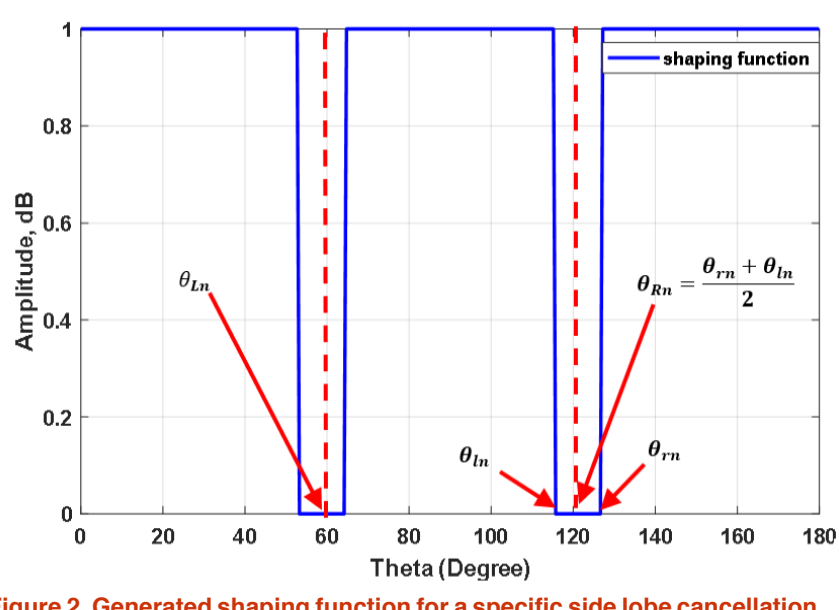

Figure 2. Generated shaping function for a specific side lobe cancellation.

\section{B. Desired Pattern Generation}

The desired pattern $A F_{d}(\theta)$ for a specific side lobe cancellation is obtained by multiplying the generated shaping function by the array pattern as follows:

$$
A F_{d}(\theta)=A F(\theta) \times f_{\text {sh }}(\theta)
$$

The desired pattern of (5) can be rewritten as:

$$
A F_{d}(\theta)=\left\{\begin{array}{cc}
0 & \theta_{l n} \leq \theta \leq \theta_{r n} \\
0 & \left(180^{\circ}-\theta_{r n}\right) \leq \theta \leq\left(180^{\circ}-\theta_{l n}\right) \\
A F(\theta) & \text { otherwise }
\end{array}\right.
$$

\section{Desired Pattern Synthesis}

To synthesize the desired pattern, it is required to determine the non-uniform elements spacing $\Delta_{i}$ and the corresponding excitation coefficients $a_{m}$. Consider that the first reference element is placed at the origin (i.e. $d_{s 1}=\Delta_{1}=$ 0 ), then $m^{\text {th }}$ element spacing with respect to the reference element is given by:

$$
d_{s m}=\sum_{i=1}^{m} \Delta_{i} \quad, m=1,2,3, \ldots M
$$

The synthesized array pattern $A F_{S}(\theta)$ can be written as:

$$
A F_{s}(\theta)=\sum_{m=1}^{M} a_{m} e^{j k d_{s m} \cos \theta}
$$

The desired and synthesized patterns should be highly matched. In this case, (7) and (9) must be equal such that:

$$
A F_{s}(\theta)=\sum_{m=1}^{M} a_{m} e^{j k d_{s m} \cos \theta}=A F_{d}(\theta)
$$

Equation (10) can be expressed in matrix form as follows:

$$
[\mathcal{G}]_{1 \times M} \times[Q]_{M \times N}=[\mathcal{D}]_{1 \times N}
$$

where $N$ is the number of samples of the desired array pattern. The number of samples is selected to be large enough to maintain the fine details of the desired pattern. $\mathcal{G}$ is a $1 \times M$ vector which contains the synthesized excitation coefficients and is written as:

$$
[\mathcal{G}]_{1 \times M}=\left[\begin{array}{lllll}
a_{1} & a_{2} & \ldots & \ldots & \ldots \\
\ldots & a_{M}
\end{array}\right]
$$

$\mathcal{Q}$ is a $M \times N$ matrix which can be expressed as:

$$
[Q]_{M \times N}=\left[\begin{array}{cccc}
e^{j k d_{S 1} \cos \theta_{1}} & e^{j k d_{s 1} \cos \theta_{2}} & \ldots & e^{j k d_{s 1} \cos \theta_{N}} \\
e^{j k d_{S 2} \cos \theta_{1}} & e^{j k d_{S 2} \cos \theta_{2}} & \ldots & e^{j k d_{S 2} \cos \theta_{N}} \\
\vdots & \vdots & & \vdots \\
e^{j k d_{S M} \cos \theta_{1}} & e^{j k d_{S M} \cos \theta_{2}} & \ldots & e^{j k d_{S M} \cos \theta_{N}}
\end{array}\right]
$$

where $\mathcal{D}$ is a $1 \times N$ vector which contains the samples of the desired pattern $A F_{d}(\theta)$ which can be expressed as:

$$
[\mathcal{D}]_{1 \times N}=\left[A F_{d}\left(\theta_{1}\right) A F_{d}\left(\theta_{2}\right) A F_{d}\left(\theta_{3}\right) \ldots \ldots A F_{d}\left(\theta_{N}\right)\right]
$$

Equation (11) has two sets of unknown parameters $a_{m}$ and $\Delta_{i}$. Because $\Delta_{i}$ is confined within the limited range $\left(0.5 \lambda \leq \Delta_{i}<\right.$ $\lambda$ ), it can be easily determined using GA optimization. While the corresponding set of excitations are obtained by solving (9) using GE technique. The GA selects the optimum values of $\Delta_{i}$ to minimize the following cost function:

$$
\begin{aligned}
\mathcal{C F}=\min & \left\{\frac{1}{\left\|S L_{s}\left(\theta_{c n}\right)\right\|-\left\|S L\left(\theta_{c n}\right)\right\|}{ }^{2}+\right. \\
& \left.\left\|\mathrm{HPBW}_{\mathrm{s}}-\mathrm{HPBW}\right\|^{2}\right\}\left.\right|_{\left\|S L_{S}\left(\theta_{c n}\right)\right\|>\left\|S L\left(\theta_{c n}\right)\right\|}
\end{aligned}
$$

where $\left\|S L_{s}\left(\theta_{c n}\right)\right\|$ is the absolute value in decibels of the side lobe of the synthesized pattern at the center angle $\theta_{c n}$. $\left\|S L\left(\theta_{c n}\right)\right\|$ is the absolute value in decibels of the side lobe of the original array pattern at the center angle $\theta_{c n} . \mathrm{HPBW}_{\mathrm{s}}$ and HPBW are the half power beamwidths of the synthesized and original array patterns, respectively.

The cost function is minimized under the constraint that $\left\|S L_{s}\left(\theta_{c n}\right)\right\|>\left\|S L\left(\theta_{c n}\right)\right\|$. Also, the cost function is minimized to provide the minimum mean square error between the half power beamwidth of the synthesized and the original array patterns. That is to achieve a synthesized main beam having the same characteristics as the main beam of the original array pattern.

The traditional GA necessitates the definition of two elements. The first is a genetic representation of the solution domain, and the second is a fitness function for assessing the solution domain. The flow chart of GA is seen in Fig. 3. The initial sets of variables, which may or may not include the optimum values, are guessed in GA. These value sets are referred to as 'chromosomes,' and this step is referred to as "initialize population". Following that, for each chromosome, the value of the objective function (fitness value) is determined. The chosen individuals are then decided from the previous generation by three processes: crossing over, mutation, and survivor selection. Step chromosomes are represented in terms of genes during the crossover. The individual's genes in the mutation are adapted to the environment, where a non-uniform mutation process can be used to randomly select a chromosome and transform it into a non-uniform random number. Individuals that have not adapted to the environment are then eliminated from the next generation. If any of the chromosomes produces the desired fitness value after mutation, the process is stopped here. Otherwise, the procedure will be replicated, beginning with the fitness assessment step and equating mutated chromosomes with a new population [15]. 


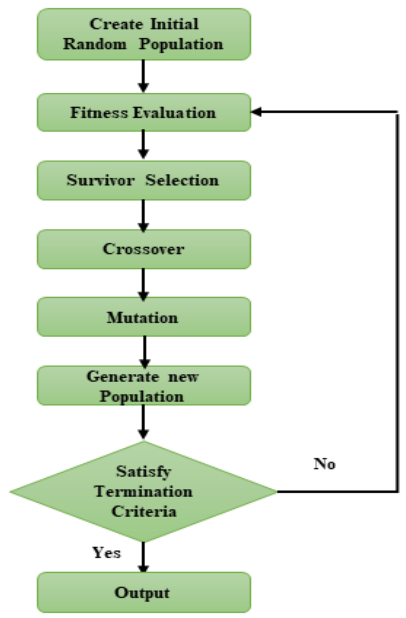

Figure 3. The genetic algorithm's flow chart.

Finally, the proposed beamforming technique can be summarized in the flowchart as shown in Fig. 4.

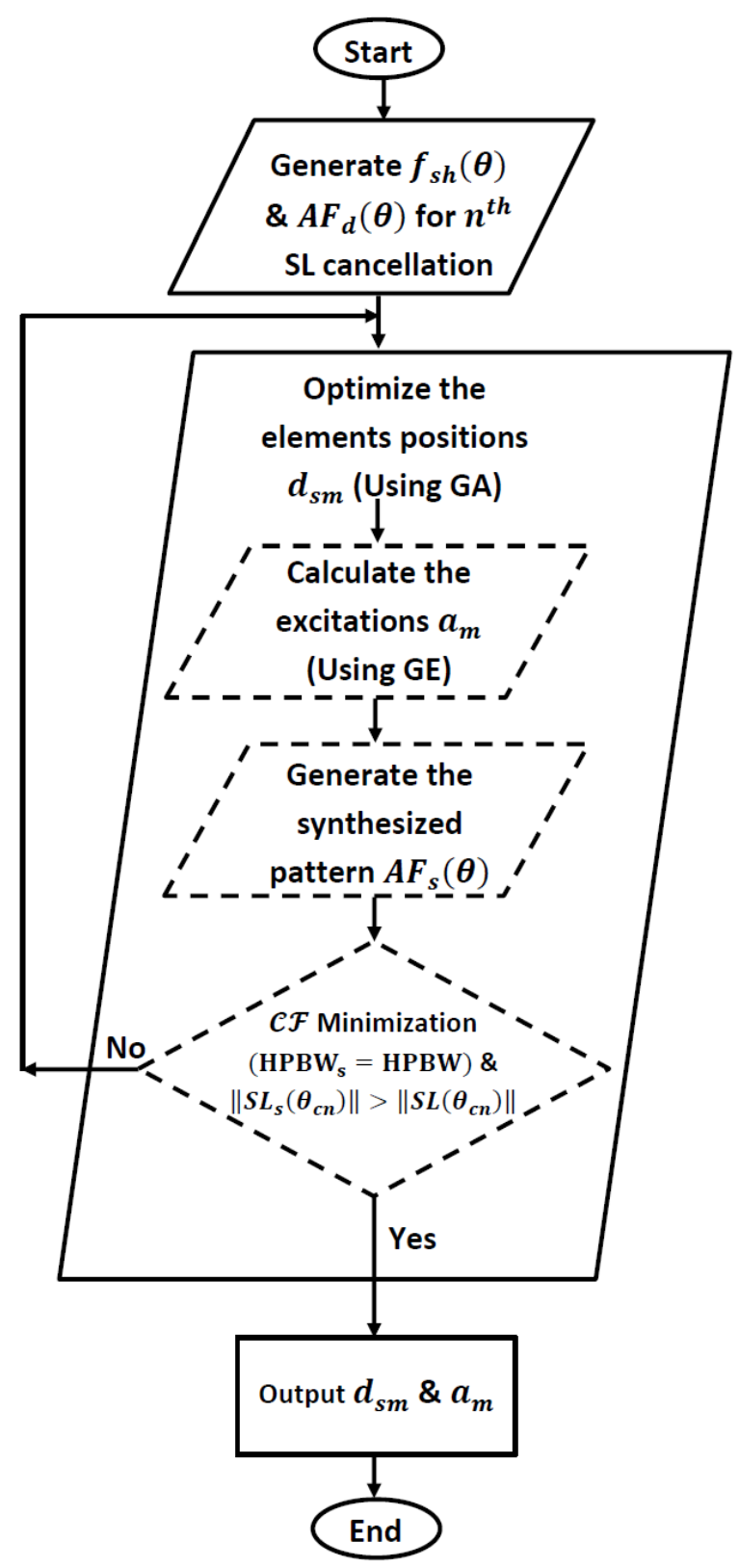

Figure 4. The flowchart of the proposed technique.

\section{Simulation Results}

In this section, two test cases are introduced to verify the effectiveness of the proposed beamforming technique. In these cases, the synthesis of two Chebychev linear antenna arrays consisting of $M=10$ and $M=20$ elements for specific SL cancellation is introduced. In the simulations, the GA optimization tool in MATLAB is used considering the setup options listed in Table 1. 


\begin{tabular}{|l|l|}
\hline \multicolumn{2}{|c|}{ GA setup options } \\
\hline Population Type & 'double vector' \\
\hline Pop Init Range & {$[2 \times 1$ double $]$} \\
\hline Population Size & 20 \\
\hline Crossover Fraction & 0.8000 \\
\hline Pareto Fraction & {[]} \\
\hline Migration Direction & 'forward' \\
\hline Migration Interval & 20 \\
\hline Migration Fraction & 0.2000 \\
\hline Generations & 400 \\
\hline Time Limit & Inf \\
\hline Fitness Limit & - Inf \\
\hline Stall Gen Limit & 50 \\
\hline Stall Time Limit & Inf \\
\hline Tot Fun & $1.000 \mathrm{e}-006$ \\
\hline Tol Con & $1.000 \mathrm{e}-6$ \\
\hline Initial population & {[]} \\
\hline Initial Scores & {[]} \\
\hline Initial Penalty & 10 \\
\hline Penalty Factor & 100 \\
\hline Plot Interval & 1 \\
\hline Creation Fcn & $@$ ga creation uniform \\
\hline Fitness Scaling Fcn & $@$ fit scaling rank \\
\hline Selection Fcn & $@$ Selection stochunif \\
\hline Crossover Fcn & $@$ Crossover scattered \\
\hline Mutation Fcn & $\{[1 \times 1]$ function_handle $[1][1]\}$ \\
\hline Distance Measure Fcn & {[]} \\
\hline Hybrid Fcn & {[]} \\
\hline & \\
\hline
\end{tabular}

Test case 1: Consider a Chebychev array consisting of $M=$ 10 antenna elements with uniform element space $d=\lambda / 2$. The array pattern has a HPBW $=13.2^{\circ}, S L L=-30 \mathrm{~dB}$ and four equal side lobes as shown in Fig. 5. It is required to perform individual side lobe cancellation while maintaining the main lobe undisrupted. The widths of the four rectangular functions are $\tau_{1}=7.45^{\circ}, \tau_{2}=11.46^{\circ}, \tau_{3}=16^{\circ}$ and $\tau_{4}=$ $36.67^{\circ}$ for the first, second, third and fourth side lobe, respectively. The synthesized array patterns for the first, second, third, and fourth side lobe are shown in Fig. 5-a, 5-b, $5-\mathrm{c}$ and 5-d, respectively. The synthesized excitation coefficients, element spacing $\Delta_{\boldsymbol{i}}$, synthesized half power beamwidth $H P B W_{s}$, minimum side lobe level $\left(S L L_{\min }\right)$, and maximum side lobe level $\left(S L L_{\max }\right)$ are listed in Table 2 . More than $25 \mathrm{~dB}$ decrements in side lobe levels are achieved after 200 GA iterations without affecting the HPBW, which remained stable and unchanged from $13.17^{\circ}$. Furthermore, as seen in Fig. 5, the main beams of the original array and the synthesized array are highly matched. The convergence curve for the first SL cancellation is shown in Fig. 6.

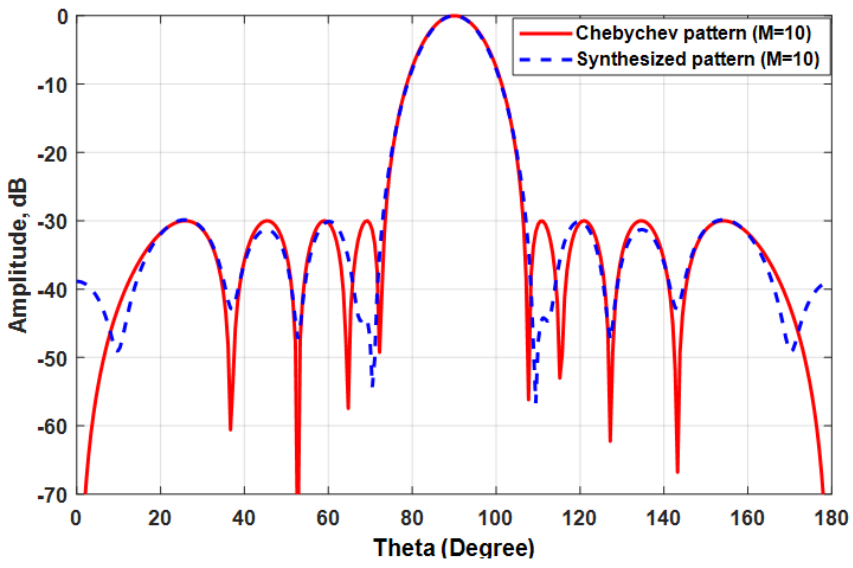

(a)

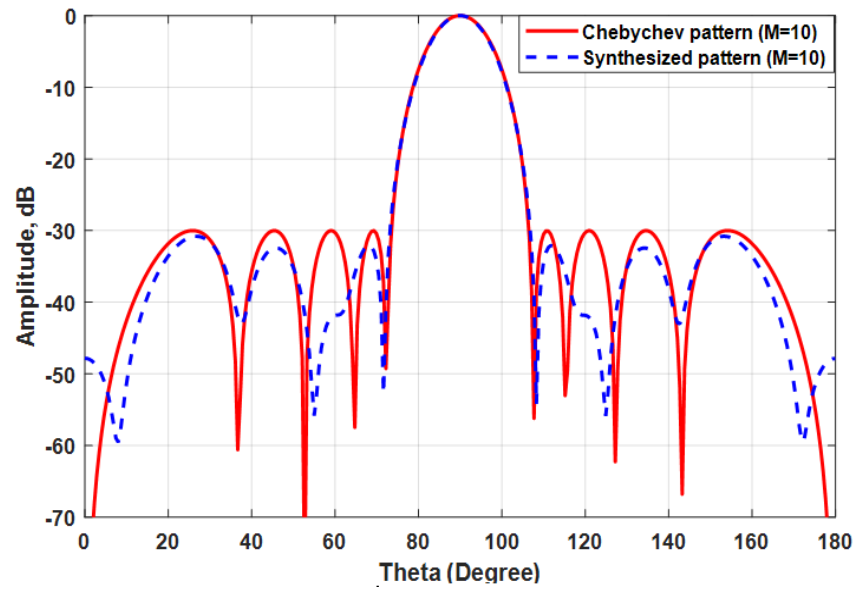

(b)

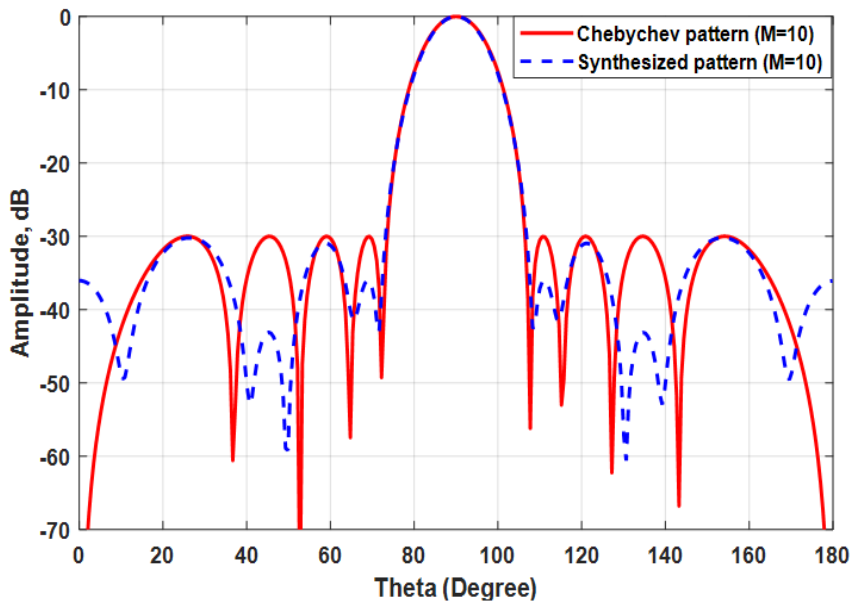

(c) 


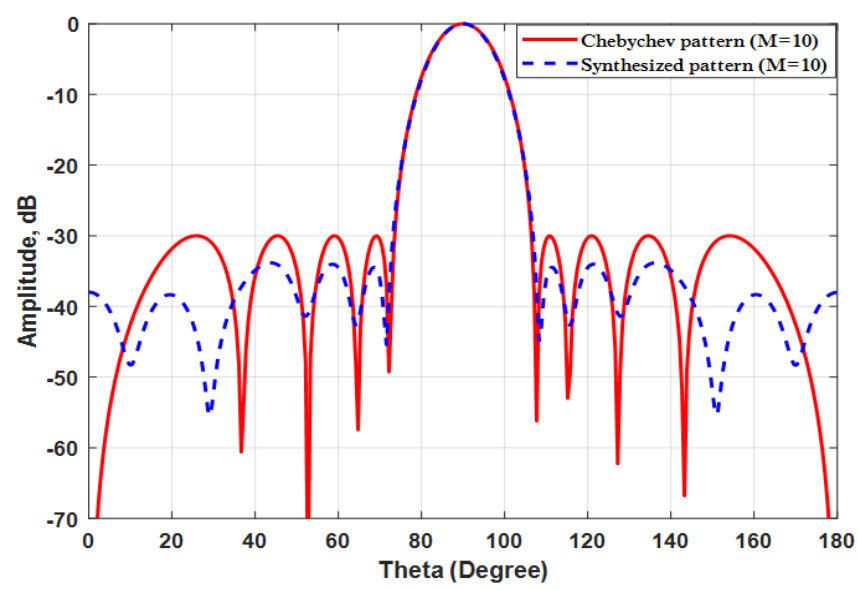

(d)

Figure 5. The synthesized Chebychev array patterns for $M=10$ elements, $S L L=-30 \mathrm{~dB}$, and $d=\lambda / 2$ for cancellation of: (a) $1^{\text {st }} \mathrm{SL}$, (b) $2^{\text {nd }} \mathrm{SL}$, (c) $3^{\text {rd }}$ $S L$, and (d) $4^{\text {th }} \mathrm{SL}$.

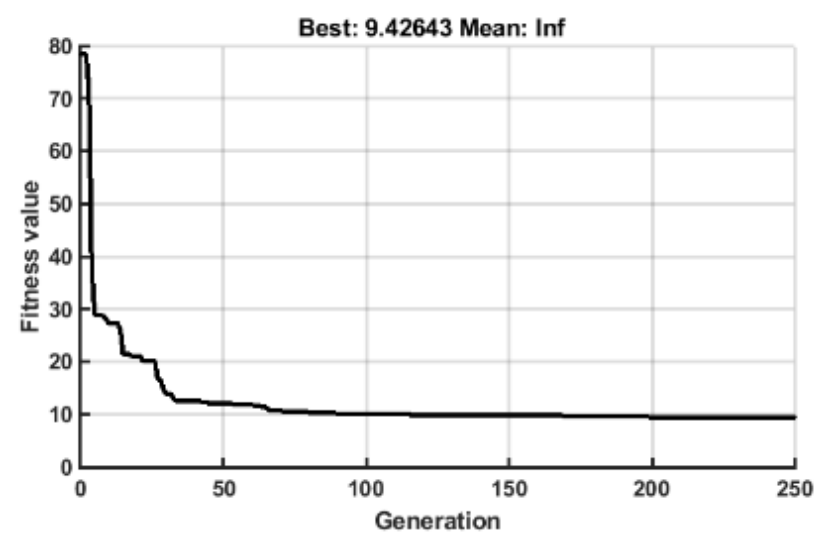

Figure 6. The convergence curve for the first SL cancellation.

Test case 2: In this case, an $M=20$ elements Chebychev antenna array with uniform element spacing $d=\lambda / 2$ is considered. The array pattern has $\mathrm{HPBW}=6.3^{\circ}, \quad S L L=$ $-30 d B$ and nine equal side lobes as shown in Fig. 7. And the null angles are also illustrated in Fig. 7.

The widths of the nine rectangular shaping functions are $\tau_{1}=0.06^{\circ}, \quad \tau_{2}=0.09^{\circ}, \quad \tau_{3}=0.1^{\circ}, \quad \tau_{4}=0.11^{\circ}, \tau_{5}=012^{\circ}$, $\tau_{6}=0.14^{\circ}, \tau_{7}=0.15^{\circ}, \tau_{8}=0.19^{\circ}$ and $\tau_{9}=0.46^{\circ}$ for the first, second, third, fourth, fifth, sixth, seventh, eight and nine SL, respectively. The corresponding synthesized array patterns are shown in Fig. 8. The synthesized excitation coefficients, $\mathrm{HPBW}_{\mathrm{s}}, S L L_{\text {min }}$, and $S L L_{\max }$ are listed in Table 3 . While the synthesized elements spacing are listed in Table 4. After $250 \mathrm{GA}$ iterations, $12 \mathrm{~dB}$ to $36.5 \mathrm{~dB}$ decrements in the side lobes levels are achieved without affecting the HPBW, which remained stable and unchanged from $6.3^{\circ}$. Furthermore, as seen in Fig. 7, the main beams of the original array and the synthesized array are highly matched.

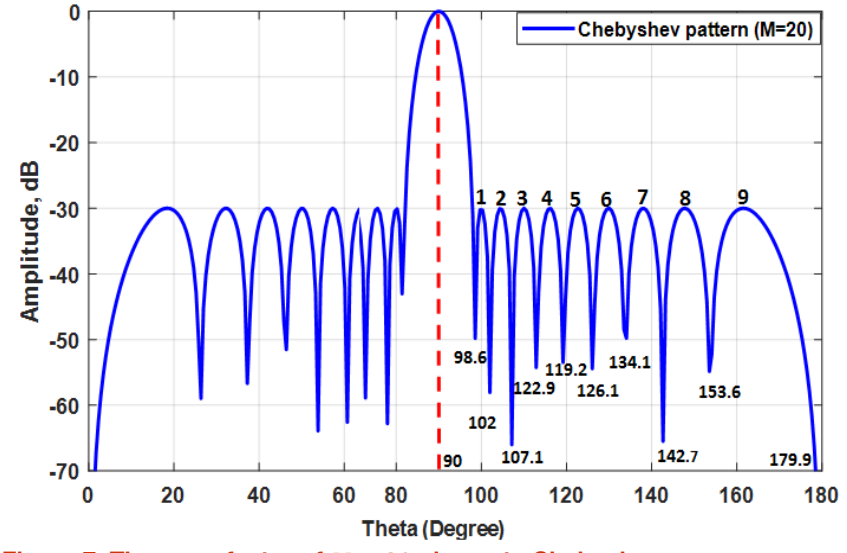

Figure 7. The array factor of $M=20$ elements Chebychev array.

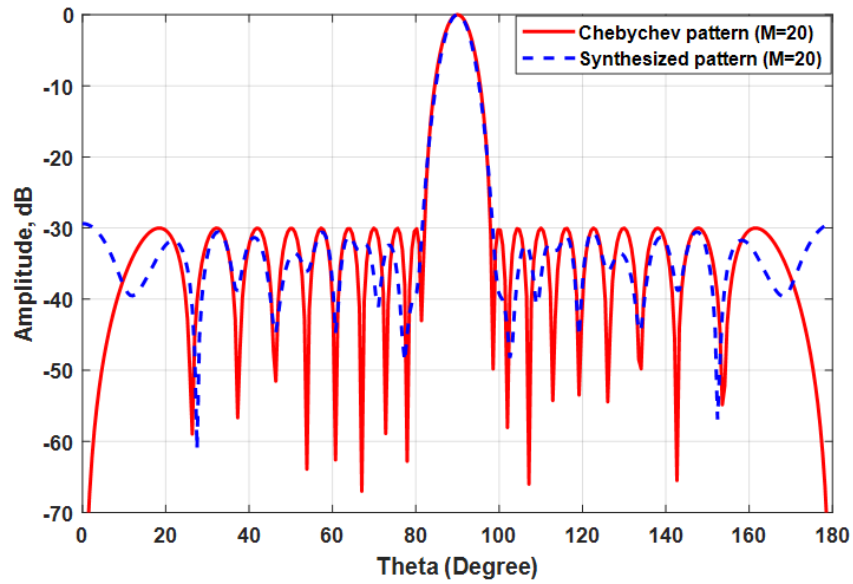

(a)

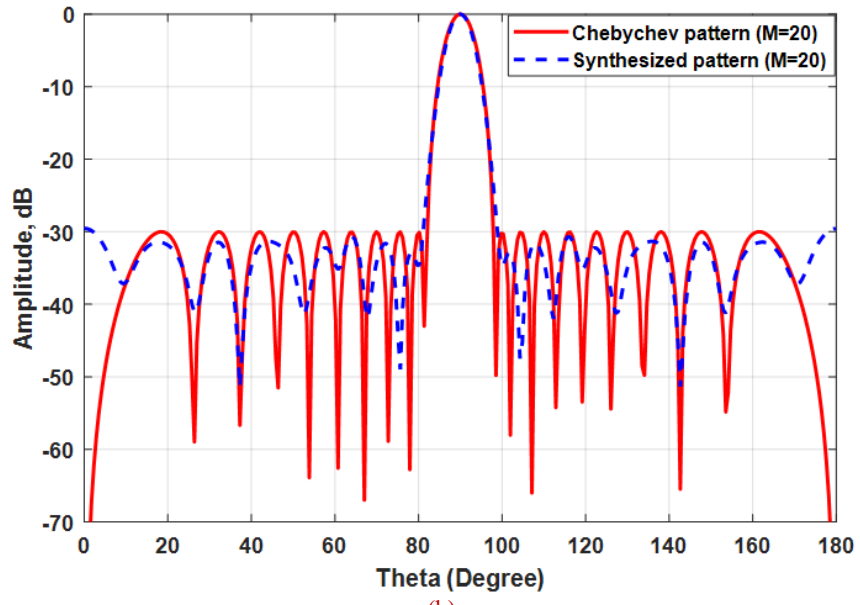

(b) 


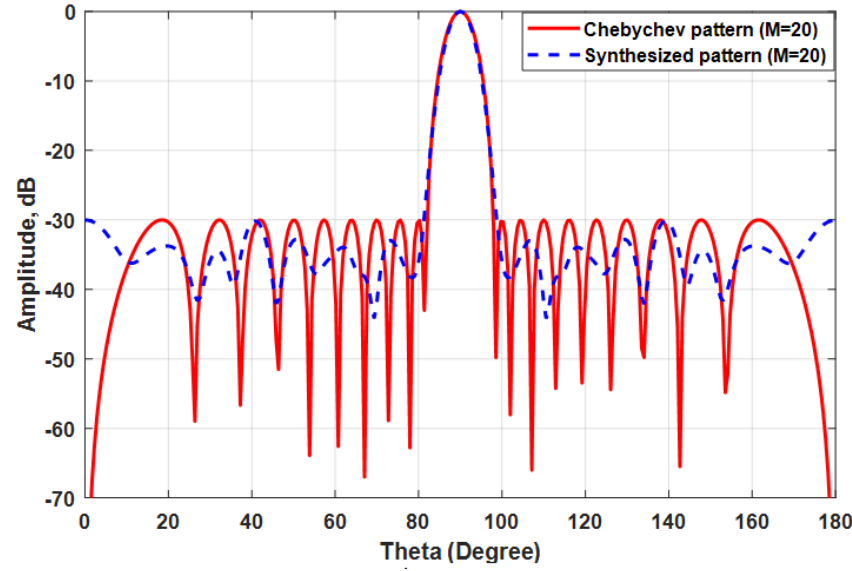

(c)

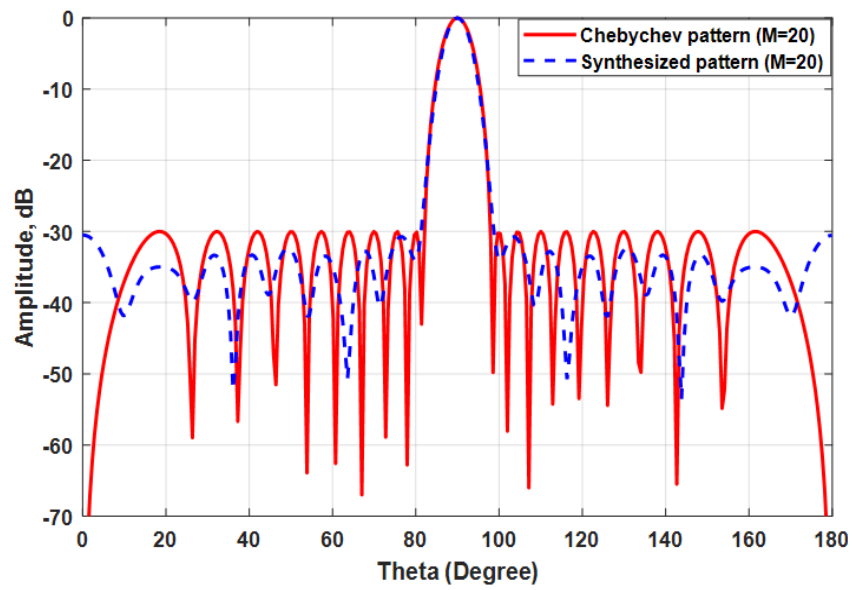

(d)

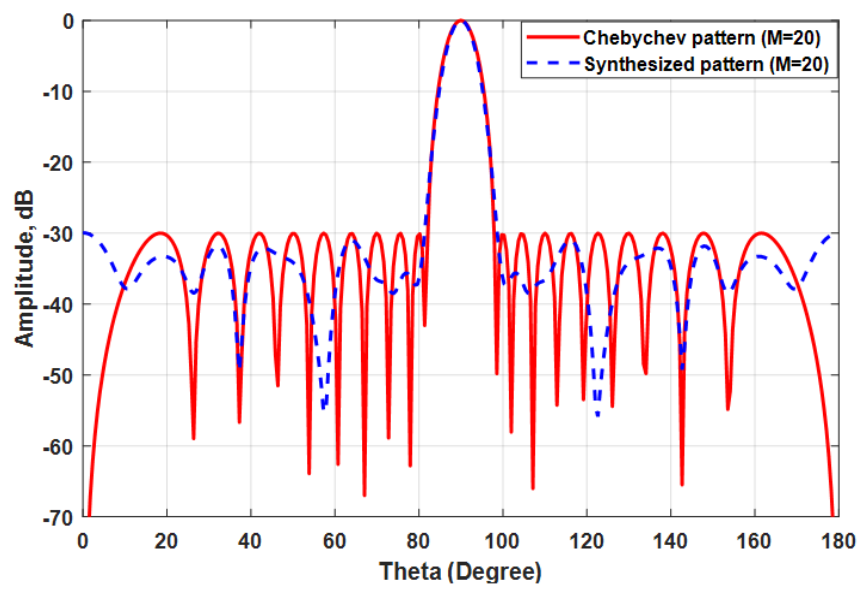

(e)

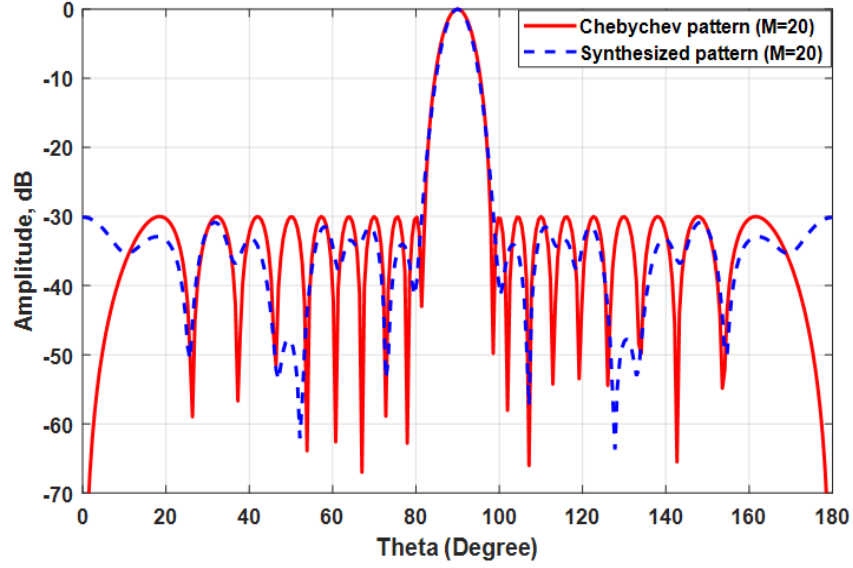

(f)

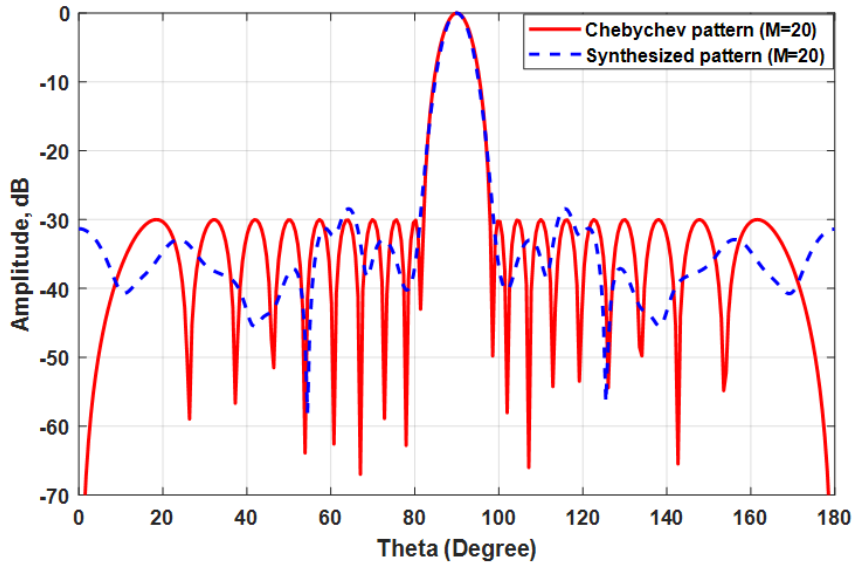

(g)

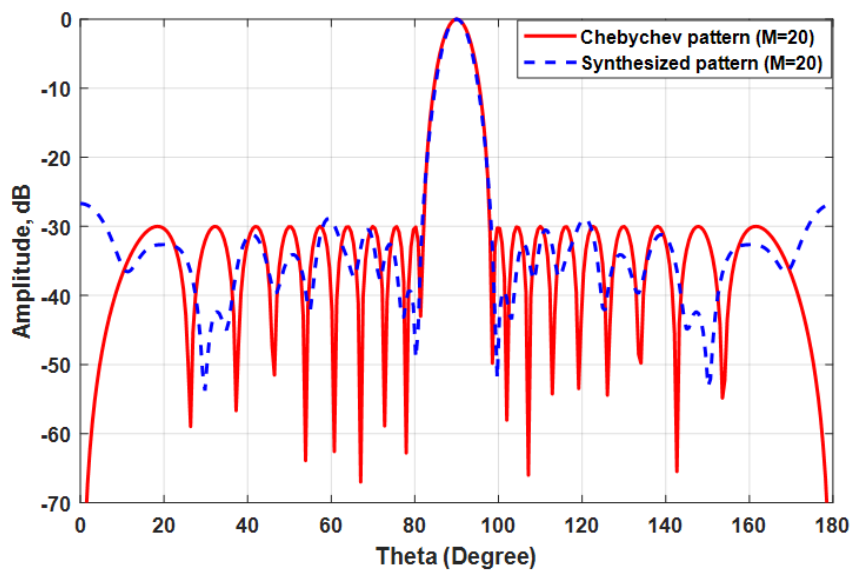

(h) 


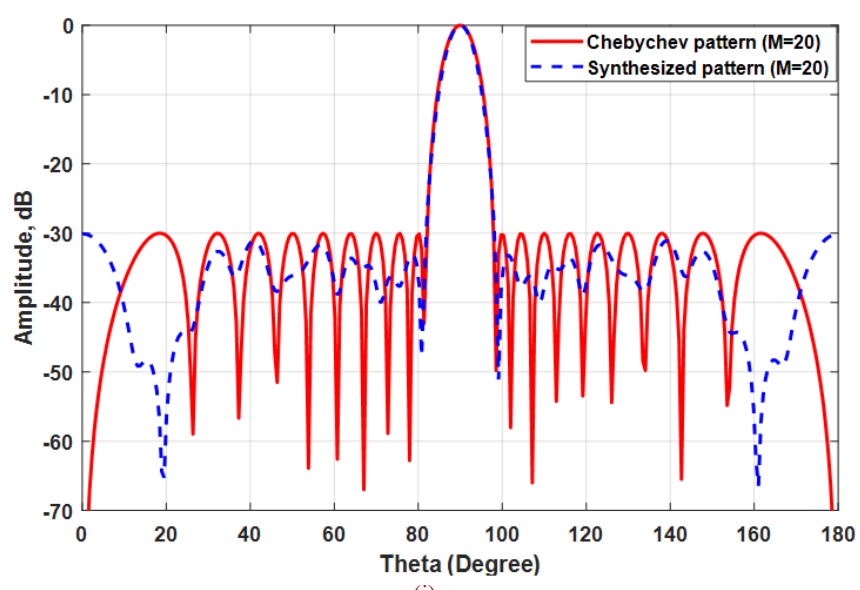

(i)

Figure 8. The synthesized Chebychev array patterns for $M=20$ elements, $S L L=-30 \mathrm{~dB}$, and $d=\lambda / 2$ for cancellation of: (a) $1^{\text {st }} \mathrm{SL}$, (b) $2^{\text {nd }} \mathrm{SL}$, (c) $3^{\text {rd }}$ SL, (d) $4^{\text {th }}$ SL, (e) $5^{\text {th }} \mathrm{SL}$, (f) $6^{\text {th }} \mathrm{SL}$, (g) $7^{\text {th }} \mathrm{SL}$, (h) $8^{\text {th }} \mathrm{SL}$, and (i) $9^{\text {th }} \mathrm{SL}$.

\section{CST Realization of the Proposed Technique}

In this section, the realistic validation of the proposed GA/GE beamforming technique is verified for real antenna elements rather than isotropic antennas. The synthesized antenna array is implemented using CST microwave studio software package using a dipole element whose dimensions, H-plane pattern, and E-plane pattern are shown in Fig. 9. The scattering parameter (reflection coefficient) $\left|S_{11}\right|$ versus frequency of the dipole antenna is shown in Fig. 10 where it has a resonance frequency $f_{o}=0.3054 \mathrm{GHz}$.

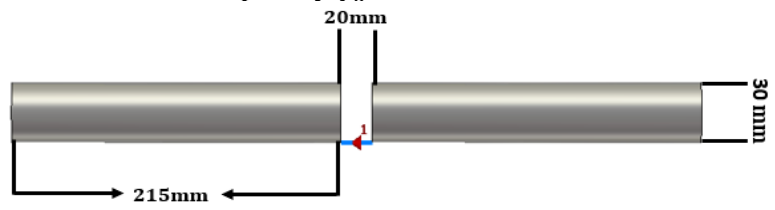

(a)

Farfield Directivity Abs (Theta $=0$ )

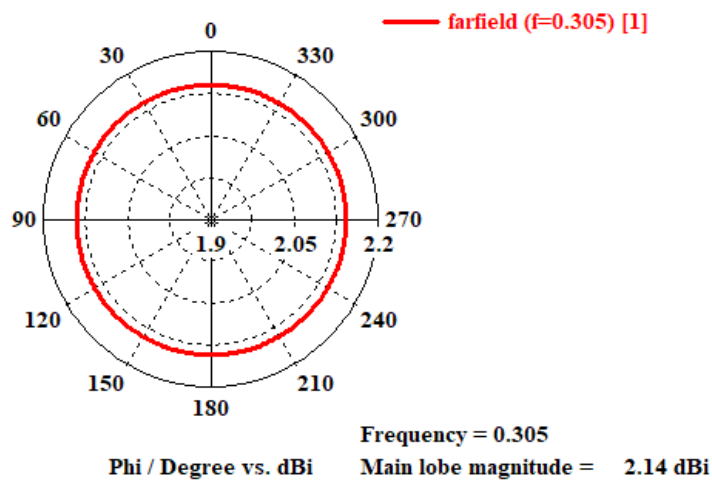

(b)
Farfield Directivity Abs (Phi=90)

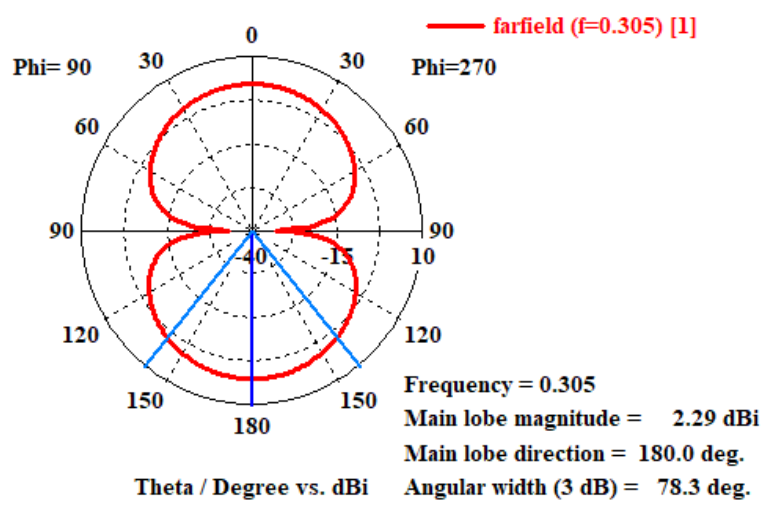

(c)

Figure 9. (a) Dipole antenna dimensions, (b) H-plane pattern, and (c) Eplane pattern of the designed dipole antenna.

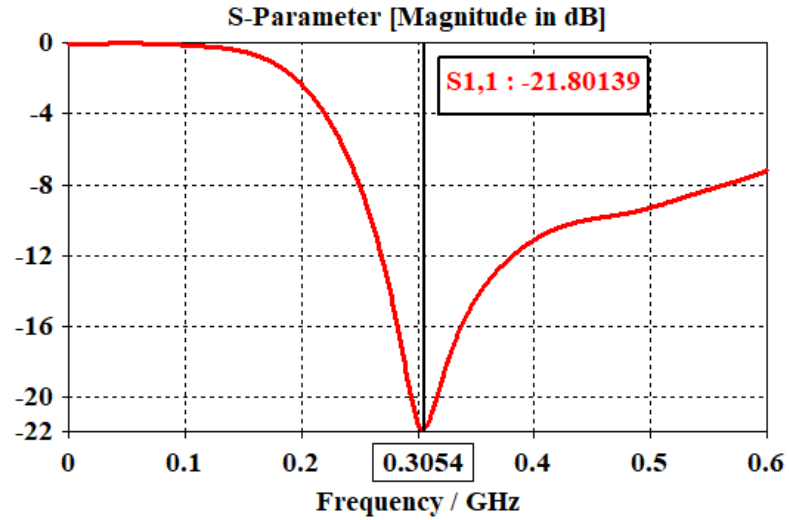

Figure 10. The scattering parameter (reflection coefficient) $\left|S_{11}\right|$ versus frequency of the designed dipole antenna.

Taking the test case of synthesizing the $\boldsymbol{M}=\mathbf{1 0}$ elements Chebychev array for the $1^{\text {st }}$ side lobe cancellation, the geometrical structure of the synthesized array using CST microwave studio is shown in Fig. 11. The dipole elements are aligned on $\mathrm{X}$-axis and oriented in the $\mathrm{Z}$ direction. The simulated $2 \mathrm{D}$ radiation pattern of the synthesized array compared to the original Chebychev array pattern is shown in Fig. 12. It is noticed that the left first SL is reduced to $-45.4 d B$ while the right first $\mathrm{SL}$ is reduced to $-47.68 \boldsymbol{d B}$. Furthermore, the two arrays main beams are perfectly coincided. Fig. 13 shows the $3 \mathrm{D}$ radiation patterns of the synthesized Chebychev array and the original Chebychev array for $1^{\text {st }} \mathrm{SL}$ cancellation using CST Microwave studio. It is noted that the $1^{\text {st }} \mathrm{SL}$ cancellation increased the gain of the synthesized array to $\mathbf{1 3} \mathbf{d B i}$ while the gain of the original array is $\mathbf{1 2 . 8} \mathbf{~} \mathbf{B} \boldsymbol{i}$.

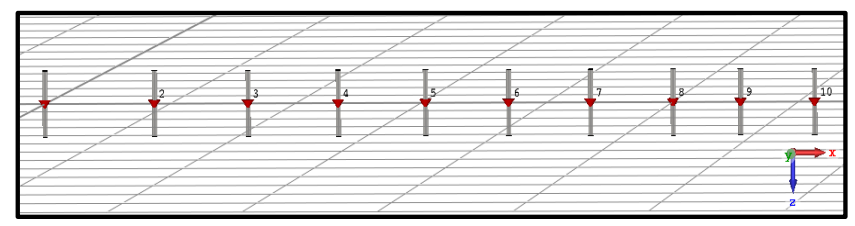

Figure 11. Geometrical structure of the $M=10$ elements synthesized array for the $4^{\text {th }}$ SL cancellation using CST microwave studio. 


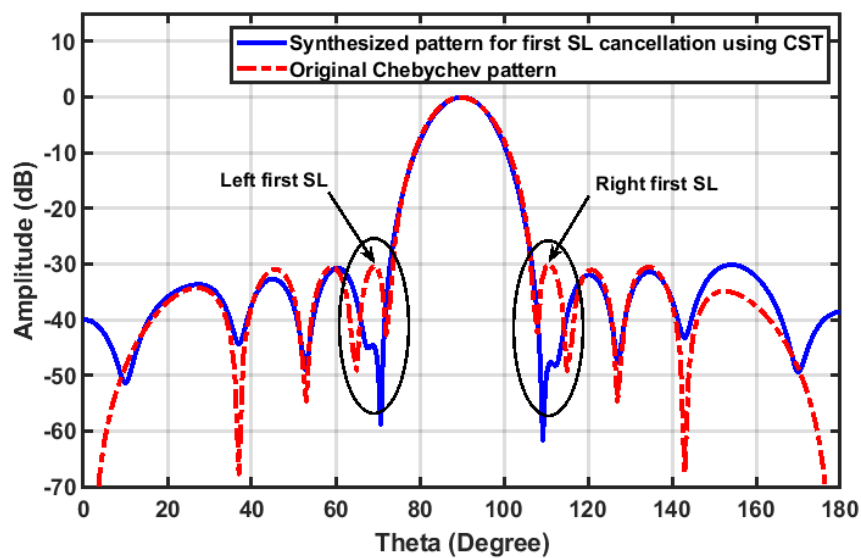

Figure 12. The original and synthesized Chebychev arrays patterns for $M=10$ elements, $S L L=-30 \mathrm{~dB}$, and $d=\lambda / 2$ for cancellation of the $1{ }^{\text {st }} \mathrm{SL}$ using CST Microwave Studio.

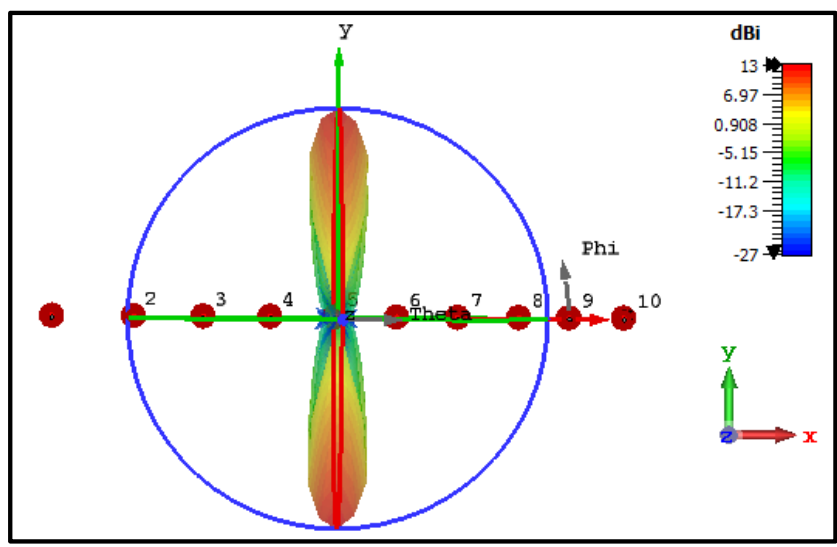

(a)

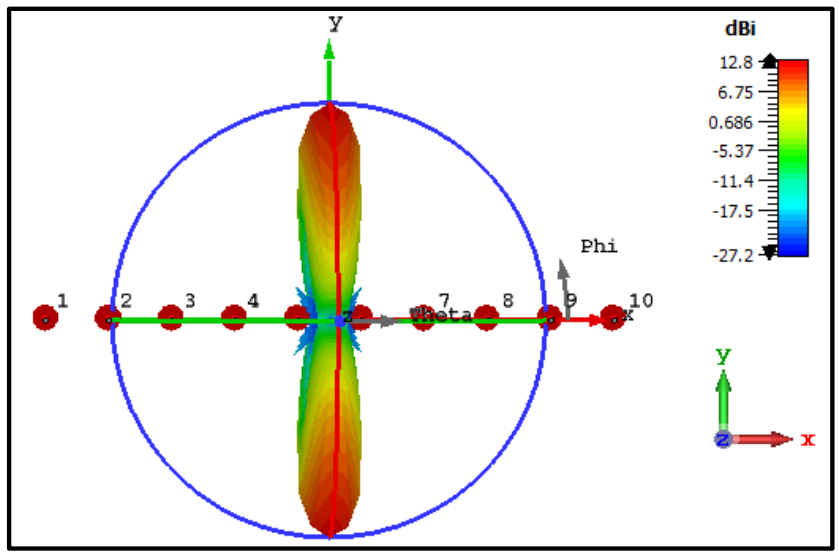

(b)

Figure 13. The 3D radiation patterns of: (a) synthesized Chebychev array and (b) original Chebychev array for $M=10$ elements, $S L L=-30 \mathrm{~dB}$, and $d=\lambda / 2$ for cancellation of the $1^{\text {st }}$ SL using CST Microwave Studio. WITH $S L L=-30 \mathrm{~dB}$.

\begin{tabular}{|c|c|c|c|c|c|c|c|c|c|}
\hline \multicolumn{2}{|c|}{ Number of SL } & \multicolumn{2}{|c|}{$1^{\text {st }} S L$} & \multicolumn{2}{|c|}{$2^{\text {nd }} S L$} & \multicolumn{2}{|c|}{$3^{\text {rd }} S L$} & \multicolumn{2}{|c|}{$4^{\text {th }} \mathrm{SL}$} \\
\hline & & $a_{m}$ & $\Delta_{i}$ & $a_{m}$ & $\Delta_{i}$ & $a_{m}$ & $\Delta_{i}$ & $a_{m}$ & $\Delta_{i}$ \\
\hline \multicolumn{2}{|c|}{$a_{1}$} & 0.1908 & 0 & 0.0615 & 0 & 0.0697 & 0 & 0.1169 & 0 \\
\hline \multicolumn{2}{|c|}{$a_{2}$} & 1.5781 & $0.8751 \lambda$ & 0.4639 & $0.6864 \lambda$ & 0.4087 & $0.5000 \lambda$ & 0.8099 & $0.8997 \lambda$ \\
\hline \multicolumn{2}{|c|}{$a_{3}$} & 3.1383 & $0.7470 \lambda$ & 1.7009 & $0.5063 \lambda$ & 1.7294 & $0.6115 \lambda$ & 2.6481 & $0.7707 \lambda$ \\
\hline \multicolumn{2}{|c|}{$a_{4}$} & 4.8571 & $0.7187 \lambda$ & 3.4970 & $0.6626 \lambda$ & 2.9068 & $0.6117 \lambda$ & 4.6789 & $0.7764 \lambda$ \\
\hline \multicolumn{2}{|c|}{$a_{5}$} & 5.4718 & $0.6948 \lambda$ & 5.0310 & $0.6898 \lambda$ & 4.3464 & $0.6117 \lambda$ & 5.9491 & $0.7634 \lambda$ \\
\hline \multicolumn{2}{|c|}{$a_{6}$} & 4.6385 & $0.6623 \lambda$ & 5.5197 & $0.7047 \lambda$ & 5.6813 & $0.6797 \lambda$ & 5.6081 & $0.7642 \lambda$ \\
\hline \multicolumn{2}{|c|}{$a_{7}$} & 3.2045 & $0.6514 \lambda$ & 4.7945 & $0.7114 \lambda$ & 5.0860 & $0.7204 \lambda$ & 3.7068 & $0.7577 \lambda$ \\
\hline \multicolumn{2}{|c|}{$a_{8}$} & 1.8120 & $0.6560 \lambda$ & 2.8711 & $0.7022 \lambda$ & 3.4467 & $0.7479 \lambda$ & 1.7498 & $0.7425 \lambda$ \\
\hline \multicolumn{2}{|c|}{$a_{9}$} & 0.4679 & $0.5366 \lambda$ & 1.2772 & $0.7227 \lambda$ & 1.6009 & $0.7674 \lambda$ & 0.1541 & $0.6107 \lambda$ \\
\hline \multicolumn{2}{|c|}{$a_{10}$} & 0.0007 & $0.5873 \lambda$ & 0.1578 & $0.8869 \lambda$ & 0.2332 & $0.8923 \lambda$ & 0.0496 & $0.7504 \lambda$ \\
\hline \multirow{2}{*}{$S L L$} & $S L L_{\min }$ & \multicolumn{2}{|c|}{$-56.7 d B$} & \multicolumn{2}{|c|}{$-55.9 d B$} & \multicolumn{2}{|c|}{$-60.6 \mathrm{~dB}$} & \multicolumn{2}{|c|}{$-55.5 d B$} \\
\hline & $S L L_{\max }$ & \multicolumn{2}{|c|}{$-40.6 \mathrm{~dB}$} & \multicolumn{2}{|c|}{$-40.6 \mathrm{~dB}$} & \multicolumn{2}{|c|}{$-43.1 \mathrm{~dB}$} & \multicolumn{2}{|c|}{$-38.3 \mathrm{~dB}$} \\
\hline \multicolumn{2}{|c|}{$\mathrm{HPBW}_{s}$} & \multicolumn{2}{|c|}{$13.17^{\circ}$} & \multicolumn{2}{|c|}{$13.17^{\circ}$} & \multicolumn{2}{|c|}{$13.17^{\circ}$} & \multicolumn{2}{|c|}{$13.17^{\circ}$} \\
\hline
\end{tabular}

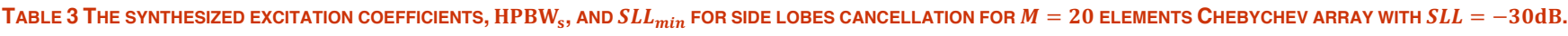

\begin{tabular}{|c|c|c|c|c|c|c|c|c|c|}
\hline $\begin{array}{c}\text { Number of } \\
\mathrm{SL}\end{array}$ & $1^{\text {st }} \mathrm{SL}$ & $2^{\text {nd }} \mathrm{SL}$ & $3^{\text {rd }} \mathrm{SL}$ & $4^{\text {th }} \mathrm{SL}$ & $5^{\text {th }} \mathrm{SL}$ & $6^{\text {th }} \mathrm{SL}$ & $7^{\text {th }} \mathrm{SL}$ & $8^{\text {th }} \mathrm{SL}$ & $9^{\text {th }} \mathrm{SL}$ \\
\hline
\end{tabular}




\begin{tabular}{|c|c|c|c|c|c|c|c|c|c|c|}
\hline & $a 1$ & 0.2696 & 0.1899 & 0.1836 & 0.2086 & 0.229 & 0.2502 & 0.2163 & 0.2572 & 0.1670 \\
\hline & $a 2$ & 0.3241 & 0.0041 & 0.3829 & 0.3535 & 0.3533 & 0.2354 & 0.4343 & 0.5084 & 0.2820 \\
\hline & 'a3 & 0.2759 & 0.2042 & 0.6726 & 0.5139 & 0.5829 & 0.4188 & 1.1136 & 0.7798 & 0.4276 \\
\hline & $a 4$ & 1.1342 & 1.0392 & 0.9228 & 1.1057 & 1.0993 & 1.1782 & 1.6006 & 1.2349 & 1.0649 \\
\hline & $a 5$ & 1.6078 & 0.8983 & 1.0540 & 1.5263 & 1.8882 & 1.7826 & 2.2417 & 1.1139 & 1.4993 \\
\hline & $a 6$ & 1.8703 & 1.8175 & 1.9134 & 1.3550 & 2.4676 & 2.3130 & 1.1770 & 2.4656 & 1.8563 \\
\hline & $a 7$ & 1.9343 & 2.9357 & 2.7989 & 2.4307 & 3.2093 & 2.2842 & 3.4338 & 3.2102 & 2.3838 \\
\hline & $a 8$ & 3.5474 & 3.8895 & 3.7032 & 3.6622 & 3.0666 & 2.7805 & 4.5088 & 3.1424 & 3.7735 \\
\hline & $a 9$ & 4.5815 & 4.7404 & 4.7410 & 4.8715 & 3.5552 & 3.8579 & 4.8302 & 2.9541 & 4.5790 \\
\hline & $a 10$ & 4.6158 & 4.5083 & 5.1859 & 5.2579 & 3.9656 & 4.1021 & 5.1400 & 4.5905 & 4.6053 \\
\hline & $a 11$ & 3.1964 & 2.2302 & 4.4956 & 4.6284 & 2.6024 & 4.1925 & 4.1358 & 5.0266 & 3.8406 \\
\hline & $a 12$ & 3.8100 & 4.2793 & 1.9601 & 1.8375 & 4.2456 & 4.6355 & 2.7601 & 4.6078 & 2.9860 \\
\hline & $a 13$ & 4.2297 & 4.0174 & 3.7154 & 4.0259 & 4.0642 & 4.1707 & 2.6064 & 3.6689 & 3.7257 \\
\hline & a14 & 3.6838 & 2.6011 & 3.3819 & 3.4444 & 3.0089 & 3.3255 & 1.8480 & 2.4576 & 3.5675 \\
\hline & a15 & 2.8248 & 2.5348 & 2.6368 & 2.7041 & 2.0184 & 2.1072 & 1.8354 & 2.1204 & 2.5948 \\
\hline & $a 16$ & 1.7462 & 2.1520 & 1.5161 & 1.5780 & 2.0494 & 1.0350 & 1.3272 & 1.7096 & 1.5788 \\
\hline & $a 17$ & 1.1231 & 1.5012 & 0.8850 & 0.6187 & 1.5381 & 1.3315 & 0.7931 & 1.3596 & 1.2898 \\
\hline & a18 & 0.6515 & 0.9573 & 0.6822 & 0.8367 & 0.9598 & 0.9260 & 0.8457 & 0.1058 & 0.6725 \\
\hline & a19 & 0.4626 & 0.5980 & 0.5181 & 0.4721 & 0.4620 & 0.4308 & 0.3494 & 0.0564 & 0.4543 \\
\hline & $a 20$ & 0.2564 & 0.2550 & 0.1683 & 0.1394 & 0.1295 & 0.2089 & 0.2579 & 0.1839 & 0.2266 \\
\hline \multirow{2}{*}{$S L L$} & $S L L_{\text {min }}$ & $-42 \mathrm{~dB}$ & $-49 \mathrm{~dB}$ & $-44.2 \mathrm{~dB}$ & $-50.8 \mathrm{~dB}$ & $-55.9 \mathrm{~dB}$ & $-63.7 \mathrm{~dB}$ & $-45.5 \mathrm{~dB}$ & $-53.8 \mathrm{~dB}$ & $-66.5 \mathrm{~dB}$ \\
\hline & $S L L_{\max }$ & $-39.1 \mathrm{~dB}$ & $-40.5 \mathrm{~dB}$ & $-36.3 \mathrm{~dB}$ & $-37.8 \mathrm{~dB}$ & $-43.7 \mathrm{~dB}$ & $-47.7 \mathrm{~dB}$ & $-41.4 \mathrm{~dB}$ & $-42.33 \mathrm{~dB}$ & $-41.7 \mathrm{~dB}$ \\
\hline \multicolumn{2}{|c|}{$\mathrm{HPBW}_{s}$} & $6.3^{\circ}$ & $6.3^{\circ}$ & $6.3^{\circ}$ & $6.3^{\circ}$ & $6.3^{\circ}$ & $6.3^{\circ}$ & $6.3^{\circ}$ & $6.3^{\circ}$ & $6.3^{\circ}$ \\
\hline
\end{tabular}

TABLE 4 The SYNTHESIZED ELEMENTS SPACING FOR SIDE LOBES CANCELLATION FOR $M=20$ ELEMENTS CHEBYCHEV ARRAY WITH $S L L=-30 \mathrm{~dB}$.

\begin{tabular}{|c|c|c|c|c|c|c|c|c|c|}
\hline $\begin{array}{c}\text { Number } \\
\text { of SL }\end{array}$ & 1 st SL & 2nd SL & 3rd SL & 4th SL & 5th SL & 6th SL & 7th SL & 8th SL & 9th SL \\
\hline$\Delta_{\mathbf{1}}$ & 0 & 0 & 0 & 0 & 0 & 0 & 0 & 0 & 0 \\
\hline$\Delta_{\mathbf{2}}$ & $0.9000 \lambda$ & $0.7567 \lambda$ & $0.9000 \lambda$ & $0.9000 \lambda$ & $0.9000 \lambda$ & $0.9000 \lambda$ & $0.8976 \lambda$ & $0.8870 \lambda$ & $0.9000 \lambda$ \\
\hline$\Delta_{\mathbf{3}}$ & $0.5079 \lambda$ & $0.5129 \lambda$ & $0.9000 \lambda$ & $0.8995 \lambda$ & $0.9000 \lambda$ & $0.9000 \lambda$ & $0.8997 \lambda$ & $0.8999 \lambda$ & $0.8996 \lambda$ \\
\hline$\Delta_{\mathbf{4}}$ & $0.5660 \lambda$ & $0.7434 \lambda$ & $0.5624 \lambda$ & $0.5008 \lambda$ & $0.5685 \lambda$ & $0.5295 \lambda$ & $0.8578 \lambda$ & $0.7835 \lambda$ & $0.5615 \lambda$ \\
\hline$\Delta_{\mathbf{5}}$ & $0.7668 \lambda$ & $0.5820 \lambda$ & $0.5152 \lambda$ & $0.6313 \lambda$ & $0.6652 \lambda$ & $0.6844 \lambda$ & $0.8504 \lambda$ & $0.5811 \lambda$ & $0.6655 \lambda$ \\
\hline$\Delta_{\mathbf{6}}$ & $0.7656 \lambda$ & $0.6239 \lambda$ & $0.6034 \lambda$ & $0.5842 \lambda$ & $0.7623 \lambda$ & $0.7727 \lambda$ & $0.5045 \lambda$ & $0.6047 \lambda$ & $0.6267 \lambda$ \\
\hline$\Delta_{\mathbf{7}}$ & $0.5439 \lambda$ & $0.7814 \lambda$ & $0.6416 \lambda$ & $0.5076 \lambda$ & $0.7990 \lambda$ & $0.6365 \lambda$ & $0.5100 \lambda$ & $0.7767 \lambda$ & $0.6163 \lambda$ \\
\hline$\Delta_{\mathbf{8}}$ & $0.6654 \lambda$ & $0.8264 \lambda$ & $0.7801 \lambda$ & $0.7941 \lambda$ & $0.6276 \lambda$ & $0.5018 \lambda$ & $0.8073 \lambda$ & $0.7459 \lambda$ & $0.6776 \lambda$ \\
\hline$\Delta_{\mathbf{9}}$ & $0.7990 \lambda$ & $0.8627 \lambda$ & $0.7999 \lambda$ & $0.8305 \lambda$ & $0.5827 \lambda$ & $0.6537 \lambda$ & $0.8661 \lambda$ & $0.5111 \lambda$ & $0.7886 \lambda$ \\
\hline$\Delta_{\mathbf{1 0}}$ & $0.7945 \lambda$ & $0.7707 \lambda$ & $0.8158 \lambda$ & $0.8393 \lambda$ & $0.6576 \lambda$ & $0.6964 \lambda$ & $0.8255 \lambda$ & $0.6615 \lambda$ & $0.7878 \lambda$ \\
\hline$\Delta_{\mathbf{1 1}}$ & $0.6590 \lambda$ & $0.5391 \lambda$ & $0.7988 \lambda$ & $0.8137 \lambda$ & $0.5030 \lambda$ & $0.6717 \lambda$ & $0.7486 \lambda$ & $0.8266 \lambda$ & $0.7530 \lambda$ \\
\hline$\Delta_{\mathbf{1 2}}$ & $0.5188 \lambda$ & $0.5276 \lambda$ & $0.5301 \lambda$ & $0.5145 \lambda$ & $0.5528 \lambda$ & $0.7284 \lambda$ & $0.6200 \lambda$ & $0.8029 \lambda$ & $0.5322 \lambda$ \\
\hline$\Delta_{\mathbf{1 3}}$ & $0.7458 \lambda$ & $0.7595 \lambda$ & $0.5039 \lambda$ & $0.5067 \lambda$ & $0.7464 \lambda$ & $0.7721 \lambda$ & $0.5432 \lambda$ & $0.7965 \lambda$ & $0.6132 \lambda$ \\
\hline$\Delta_{\mathbf{1 4}}$ & $0.8310 \lambda$ & $0.6506 \lambda$ & $0.8069 \lambda$ & $0.8374 \lambda$ & $0.7705 \lambda$ & $0.8212 \lambda$ & $0.5242 \lambda$ & $0.6623 \lambda$ & $0.7720 \lambda$ \\
\hline$\Delta_{\mathbf{1 5}}$ & $0.8119 \lambda$ & $0.5329 \lambda$ & $0.8480 \lambda$ & $0.8680 \lambda$ & $0.5713 \lambda$ & $0.8008 \lambda$ & $0.5113 \lambda$ & $0.6409 \lambda$ & $0.7298 \lambda$ \\
\hline$\Delta_{\mathbf{1 6}}$ & $0.7548 \lambda$ & $0.7952 \lambda$ & $0.7030 \lambda$ & $0.7956 \lambda$ & $0.6612 \lambda$ & $0.5068 \lambda$ & $0.7381 \lambda$ & $0.7631 \lambda$ & $0.6853 \lambda$ \\
\hline$\Delta_{\mathbf{1 7}}$ & $0.6987 \lambda$ & $0.8758 \lambda$ & $0.5950 \lambda$ & $0.5223 \lambda$ & $0.7972 \lambda$ & $0.6237 \lambda$ & $0.5062 \lambda$ & $0.8067 \lambda$ & $0.7070 \lambda$ \\
\hline$\Delta_{\mathbf{1 8}}$ & $0.6298 \lambda$ & $0.8418 \lambda$ & $0.6320 \lambda$ & $0.5135 \lambda$ & $0.8076 \lambda$ & $0.7437 \lambda$ & $0.5212 \lambda$ & $0.7850 \lambda$ & $0.7752 \lambda$ \\
\hline$\Delta_{\mathbf{1 9}}$ & $0.8889 \lambda$ & $0.9000 \lambda$ & $0.8810 \lambda$ & $0.8999 \lambda$ & $0.8996 \lambda$ & $0.9000 \lambda$ & $0.9000 \lambda$ & $0.5192 \lambda$ & $0.8999 \lambda$ \\
\hline$\Delta_{\mathbf{2 0}}$ & $0.8672 \lambda$ & $0.8999 \lambda$ & $0.8387 \lambda$ & $0.8958 \lambda$ & $0.8917 \lambda$ & $0.8974 \lambda$ & $0.8949 \lambda$ & $0.8324 \lambda$ & $0.9000 \lambda$ \\
\hline
\end{tabular}


2.

\section{CONCLUSION}

The proposed hybrid GA/GE beamforming technique is introduced in this paper using a combination of GA optimization and GE. It is used in linear antenna arrays for precise side lobe cancellation. This reduces the received interference in the appropriate directions, which improves the receiver sensitivity. The GE determines the excitation coefficients of the antenna elements, while the GA determines the optimum element positions in order to synthesize an antenna array having a pattern where a specified side lobe is minimized. To verify the effectiveness of the proposed beamforming technique, two Chebychev linear antenna arrays consisting of $M=10$ and $M=20$ elements with $S L L=-30 \mathrm{~dB}$ are beamformed for specific SL cancellation. The proposed technique provided significant SLL reductions without affecting the HPBW of the array and the main beams of the original arrays and the synthesized arrays are highly matched. For $M=10$ elements array, more than $25 \mathrm{~dB}$ decrements in side lobe levels are achieved after $200 \mathrm{GA}$ iterations. While for $M=$ 20 elements array, $12 \mathrm{~dB}$ to $36.5 \mathrm{~dB}$ decrements in the side lobes levels are achieved after 250 GA iterations. Furthermore, the feasibility of realistic validations of synthesized arrays is demonstrated using the computer simulation technology (CST) microwave studio software package. In addition, it can be concluded that SL cancellation increases the realized array gain as in the case of $1^{\text {st }}$ SL cancellation of the $M=10$ elements Chebychev array, where the gain of the synthesized array is increased to $13 \mathrm{dBi}$ while the gain of the original array is $12.8 \mathrm{dBi}$.

\section{References}

[1] M. GH. Alijani1, M. H. Neshati1, and M. Boozari, "Side Lobe Level Reduction of Any Type of Linear Equally Spaced Array Using the Method of Convolution", Progress In Electromagnetics Research Letters, vol. 66, pp. 79-84, 2017.

[2] S U Rahman, C. Qunsheng, A. M. Mansoor, and K. Hisham,"Analysis of Linear Antenna Array for minimum Side Lobe Level, Half Power Beamwidth, and Nulls control using PSO", J. Microw. Optoelectron. Electromagn., vol.16, no. 2, pp. 577-591, 2017.

[3] U. Singh, R. Salgotra, "Synthesis of linear antenna array using flower pollination algorithm", Neural Computing and Applications, vol. 29, no. 2, pp.435-445, 2018.

[4] G. Sun , Y. Liu, H. Li, S. Liang, A. Wang and B. Li," An Antenna Array Sidelobe Level Reduction Approach through Invasive Weed Optimization ", International Journal of Antennas and Propagation, vol. 4, pp. 1-16, 2018.

[5] M. Hesari and A. Ebrahimzadeh," Introducing Deeper Nulls and Reduction of Side-Lobe Level in Linear and Non-Uniform Planar Antenna Arrays Using Gravitational Search Algorithm", Progress In Electromagnetics Research B, vol. 73, pp. 131-145, 2017.

[6] A. M. Ismaiel, S. Elsaidy, Y. Albagory, and H. A. Atallah," Performance improvement of high altitude platform using concentric circular antenna array based on particle swarm optimization." AEUInternational Journal of Electronics and Communications, vol. 91 pp.85-90, 2018.

[7] A. S. Zare, S. Baghaiee, "Application of Ant Colony Optimization Algorithm to Pattern Synthesis of Uniform Circular Antenna
Array." Applied Computational Electromagnetics Society Journal, vol. 30, no. 8, 2015.

[8] Zhang, L., Y. C. Jiao, B. Chen, and F. S. Zhang, "Synthesis of linear aperiodic arrays using a self-adaptive hybrid differential evolution algorithm," Microwave and Antennas Propag., vol. 5, pp.1524-1528, 2011.

[9] H. Rezagholizadeh, D. Gharavian, "A Thinning Method of Linear and Planar Array Antennas To Reduce SLL of Radiation Pattern By GWO And ICA Algorithms", AUT Journal of Electrical Engineering, vol. 50, no. 2, pp. 135-140, 2018.

[10] M. Khodier," Comprehensive study of linear antenna array optimisation using the cuckoo search algorithm", IET Microwaves, Antennas \& Propagation, vol. 13, no. 9, pp. 1325 - 1333, 2019.

[11] I. P. Gravas, Z. D. Zaharis, T. V. Yioultsis, P. I. Lazaridis and T. D. Xenos, "Adaptive Beamforming With Sidelobe Suppression by Placing Extra Radiation Pattern Nulls," in IEEE Transactions on Antennas and Propagation, vol. 67, no. 6, pp. 3853-3862, 2019.

[12] S. Veisee and S. Asadi, A Modified Unequally Spaced Array Antenna Synthesis Method for Side Lobe Reduction. Radio engineering, vol. 26, no. 1, pp. 114-119, 2017.

[13] A. Sharma, S. Mathur," A novel adaptive beamforming with reduced side lobe level using GSA", COMPEL- The international journal for computation and mathematics in electrical and electronic engineering, vol. 37, no. 6, pp. 2263-2278, 2018.

[14] Balanis, Constantine A," Antenna theory: analysis and design', John wiley \& sons, 2015.

[15] V. Mallawaarachchi , 'Introduction to genetic algorithms-including example code"’., Towards Data Science, vol. 8, no. 7, 2017. 